\title{
El espacio rural en la enseñanza secundaria: hacia un enfoque más integrador y dinámico
}

\author{
José SÁnChez SÁNChez *
}

\begin{abstract}
RESUMEN
ABSTRACT

La Geografía debe aprovechar al máximo la capacidad educativa que encierran sus contenidos disciplinares.

Aplicando este principio a la enseñanza del espacio rural, en este artículo se propone que se aplique un concepto más integrador y dinámico

que haga más atractivo y eficaz el aprendizaje de conceptos, procesos y problemas relacionados con la evolución y diferenciación del mundo rural. Para fijar conceptos, potenciar el método indagatorio y desarrollar la sensibilidad de los alumnos es de gran utilidad la interpretación del paisaje rural que constituye un excelente recurso didáctico.

PALABRAS CLAVE Espacio rural. Paisaje rural. Educación geográfica.

Geography must make the most of the educational capacity of its disciplinar contents. Applying this principle to the teaching of the rural space, this article proposes a more integrating and dynamic concept that will make more attractive and effective the learning of concepts, processes and problems related to the evolution and differentiation of the rural world. The interpretation of the rural landscape, which forms an excellent didactic resource, is very useful to fix concepts, to foster a methodology based on investigation and to develop the sensitivity of students.

\section{KEY WORDS}

Rural space. Rural landscape. Geographic education.
\end{abstract}

* Departamento de Geografía. U.N.E.D., Madrid. 


\section{INTRODUCCIÓN}

La capacidad educativa que tiene la Geografía está fuera de toda duda. El ámbito de conocimiento objeto de su estudio se halla íntimamente relacionado con la vida de las personas y de las sociedades. Por ello es una ciencia social; por ello, también, en todos los países tiene un lugar asegurado, más o menos relevante, entre las disciplinas que integran el currículo de las enseñanzas básicas, reconociendo así explícitamente el gran valor educativo que encierran sus contenidos.

Otra cuestión es la forma en que los profesores de Geografía afrontan este reto que la disciplina contrae con la sociedad. No siempre se enseñan los contenidos más adecuados, ni se trabajan en el aula los conceptos fundamentales, ni se estructuran de la forma más conveniente. A veces se olvida la visión integradora que debe dominar en la enseñanza de la Geografía, para perderse en análisis y descripciones pormenorizadas que anulan o desvirtúan el sentido globalizador. Por ello, cuando se enseña Geografía en la etapa secundaria, es necesario tener siempre presente que el espacio geográfico es fundamentalmente integrador y dinámico, formado por "un conjunto indisociable de sistemas de objetos y acciones de carácter dinámico", como lo define Milton Santos (1996).

Además, el compromiso de la Geografía con la sociedad exige que potenciemos un tipo de aprendizaje que tenga como prioridad formar ciudadanos capaces de imaginar y adaptarse a las situaciones cambiantes de nuestro mundo (Estébanez, J., 1996); ciudadanos que, además de estar bien informados, sean críticos con las realidades y sensibles a los problemas. En definitiva, la Geografia tiene que contribuir desde su ámbito de conocimiento a potenciar la razón, objetivo que, como propone F. Sabater (1998), debe ser fundamental en la educación del siglo XXI.

A ello quieren contribuir estas reflexiones en torno al espacio rural que, con el fin de aprovechar al máximo la capacidad eductiva de su aprendizaje, debe basarse, como toda la Geografía, en un concepto integrador y dinámico del espacio.

\section{EL ESTUDIO DEL ESPACIO RURAL Y SU VALOR EDUCATIVO}

En la Geografía tradicional el estudio de las estructuras y de los paisajes agrarios ha sido objeto de una atención destacada por parte de muchos geógrafos. Esto no es de extrañar, ya que hasta los años cincuenta, y hasta los setenta en España, hemos vivido en un mundo mayoritaria- 
mente agrario, no sólo por la extensión superficial que ocupaba la actividad agropecuaria y por la alta proporción de población activa agraria y población rural, sino también por la gran significación económica de esta actividad y por la atracción que las estructuras y paisajes agrarios ejercían en la Geografía descriptiva regional, como criterio de diferenciación entre las regiones.

Medio rural y agricultura se yuxtaponían y, al ser la agricultura y la ganadería los elementos casi exclusivos que se trataban, se impuso la denominación de agraria a esta parte de la Geografía. El campo era así un ámbito bien delimitado frente a la ciudad y al espacio industrial; un mundo heredado del pasado que imprimía carácter a las regiones y comarcas, pero que era concebido como un espacio estático y atrasado frente a la ciudad y la industria.

Este concepto es el que ha dominado hasta hace poco en la mayor parte de los libros de texto de la enseñanza básica y secundaria. Y todavía sigue presente en muchos de ellos, con el agravante de que poco a poco va reduciendo su extensión en los programas, debido a la pérdida de interés de los aspectos y problemas agrarios en una sociedad casi exclusivamente urbana que pretende convertirlo todo en espacio urbano o urbanizado. A ello contribuye el hecho de que cada vez haya menos agricultores y menos población rural; que cada vez la agricultura tenga menos peso en la economía y que los geógrafos hayan perdido interés por el estudio del paisaje. Esta concepción es la que se ha plasmado en el Real Decreto de contenidos mínimos para la Geografía de segundo curso de Bachillerato (Real Decreto 1178/1992 y Real Decreto 1179/1992, de 2 de octubre; anexos I y II, en suplemento al BOE núm. 253 de 21 de octubre de 1992).

Frente a esta decadencia de la Geografía Agraria está adquiriendo un notable interés el estudio del espacio rural, cuyo concepto, más integrador y dinámico, está ya muy extendido por otros países europeos (Gilg, A.W., 1985; Kayser, B., 1990; Bonnamour, J., 1995) y se está difundiendo ampliamente por el ámbito universitario de la Geografía española (Estébanez, J., 1986; Molinero, F. y Alario, M., 1994; García Ramón, Mª.D., 1995; Ramos Real, E. y Cruz Villalón, J., 1995; Frutos, 1995; Sánchez, J., 1996; Cabero, V., 1998). Sin embargo, no acaba de llegar a los programas de Geografía de la enseñanza secundaria. Desde luego, ninguna de las normas reguladoras de esta enseñanza secundaria contempla este enfoque del espacio rural.

$Y$, sin embargo, el valor educativo del estudio del medio rural es extraordinario, debido a que el concepto de espacio en el que se basa le permite integrar diversos ámbitos, dotándolo de una gran riqueza de conteni- 
dos y le obliga a plantear de forma dialéctica las acciones y reacciones derivadas de los numerosos actores naturales y humanos y de políticas de intervención que tratan de resolver los problemas y conflictos que afectan a las sociedades rurales.

Cuadro 1. Ámbitos de contenidos del espacio rural

EL ESPACIO RURAL Y SU RIQUEZA EN CONTENIDOS TEMÁTICOS

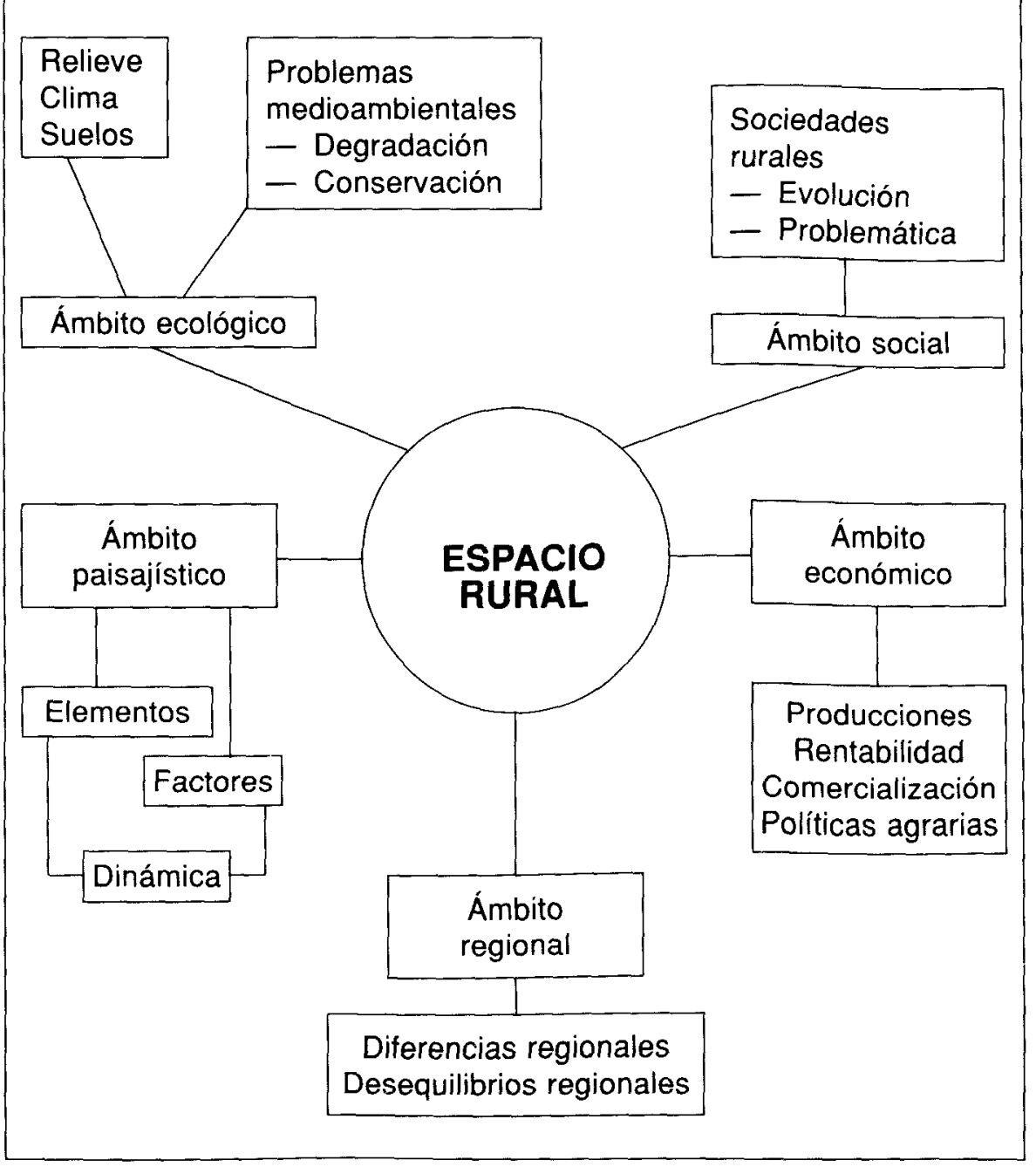


Varios son los ámbitos temáticos que se ven implicados en el estudio del espacio rural: el ecológico, el social, el económico, el paisajístico y el regional. En el ámbito ecológico se deben integrar los elementos y factores naturales que intervienen en la génesis y evolución de los espacios rurales, asi como los problemas medioambientales derivados de las distintas acciones humanas.

El ámbito social incluye la atención a los problemas de las sociedades rurales, relacionados tanto con su propia evolución demográfica, como con la estructura de los grupos sociales y con las repercusiones que sobre ellas provocan la evolución de la economía y las políticas agrarias y rurales. El ámbito económico contempla el análisis y localización de las producciones y los problemas de su rentabilidad y comercialización, así como el estudio de las políticas agrarias y rurales y su incidencia en el uso del suelo, la evolución de los cultivos, las nuevas actividades económicas y el desarrollo de la población rural.

La dimensión regional del espacio rural se refiere a la repercusión que las producciones, las estructuras de propiedad y explotación y las transformaciones de la economía y sociedad rurales tienen en el proceso de diferenciación regional y en la génesis y evolución de los desequilibrios regionales. Por último, el ámbito paisajístico tiene una gran importancia en el estudio del espacio rural y sobre todo un gran valor didáctico en los niveles de enseñanza primaria y secundaria, por cuanto que el paisaje rural representa una condensación de naturaleza, historia y tranformaciones recientes y supone, como todo paisaje geográfico, un excelente campo de educación geográfica (Sánchez, J., 1994; Gómez Ortiz, A., 1996; Pena, R., 1996).

El estudio del medio rural, tratado con este enfoque integrador y dinámico, permite trabajar en el aula un gran número de conceptos, procesos y problemas que, no sólo enriquecen los conocimientos de los alumnos, sino que también les ayudan a ponerse en contacto con el mundo real en el que viven y a desarrollar sensibilidades hacia los problemas que afectan a otros grupos sociales y a otros espacios geográficos. En los cuadros 2 y 3 se recoge una selección de conceptos, procesos y problemas que se pueden trabajar con el estudio del espacio rural.

\section{DEL SECTOR AGRARIO AL ESPACIO RURAL}

El primer paso que hay que dar para dotar de mayor interés al estudio de la Geografía rural y potenciar su valor educativo, dando entrada a ese gran número de conceptos, procesos y problemas recogidos en los cua- 
dros 2 y 3 , es introducir el concepto de espacio rural y estructurar los contenidos del mismo con una lógica integradora, procurando que tengan su lugar adecuado todos y cada uno de los ámbitos que debe contemplar el espacio rural, recogidos en el cuadro $n^{0}-1$.

Cuadro 2. El estudio del espacio rural (A)

\begin{tabular}{|lll|}
\hline \multicolumn{2}{c|}{ Conceptos } \\
\hline censo agrario & hortalizas & S.A.U. (Superficie Agraria Útil) \\
espacio natural & barbecho & UTA (Unidades de Trabajo Anual) \\
espacio rural & policultivo/monocultivo) & UDE (Unidad de Dimensión \\
paisaje agrario & agricultura tradicional & Europea) \\
agricultura/ganadería & agricultura intensiva/extensiva & FEOGA (Fondo Europeo de \\
parcela & agricultura de autoconsumo & Orientación y Garantía Agraria) \\
explotación agraria & agricultura comercial & competitividad \\
propiedad & agricultura productivista & pluriactividad \\
arrendamiento/aparceria & cultivo bajo plástico & productividad \\
habitat rural & agricultura postproductivista & desarrollo rural \\
estructura agraria & agricultura biológica & PAC (Política Agraria Común) \\
secano/regadio & enarenado & cuotas \\
rotación de cultivos & invernadero & subvenciones \\
leguminosas & sociedad cooperativa & política medioambiental \\
cultivos herbáceos & política agraria & LEADER (Relaciones entre \\
cultivos industriales & concentración parcelaria & actividades de desarrollo de la \\
cultivos forrajeros & & economía rural) \\
\hline
\end{tabular}

El esquema conceptual del cuadro no 4 está pensado para el estudio del medio rural en la enseñanza secundaria y resume la estructura de los contenidos fundamentales.

Lo primero que se quiere resaltar es la necesidad de pasar de unos contenidos reducidos a lo que en muchos libros se denomina sector agrario, con enfoque claramente economicista y poco geográfico, a unos conceptos más amplios, todos ellos integrados en el concepto de espacio rural.

Las estructuras agrarias (ocupación del suelo, cultivos, ganadería, propiedad, explotaciones, parcelas y hábitat) deben disponer de un lugar destacado y preeminente, ya que siguen ocupando la mayor parte del territorio calificado como rural y en la mayor parte de la tierra la actividad agraria es la más importante y la que deja las huellas más extensas y visibles en el paisaje rural. 
Cuadro 3. El estudio del espacio rural (B)

\begin{tabular}{|c|c|}
\hline \multicolumn{2}{|r|}{ Procesos } \\
\hline & $\begin{array}{l}\text { despoblación/emigración/éxodo rural } \\
\text { deterioro de las estructuras demográficas } \\
\text { modernización de las estructuras agrarias } \\
\text { deterioro medioambiental } \\
\text { transformaciones de los sistemas ganaderos } \\
\text { reducción del número de explotaciones } \\
\text { transición de una agricultura a otra } \\
\text { aparición de nuevas funciones } \\
\text { reactivación del mundo rural } \\
\text { transformación de paisajes }\end{array}$ \\
\hline \multicolumn{2}{|r|}{ Problemas } \\
\hline demográficos: & $\begin{array}{l}\text { escasa densidad de población } \\
\text { envejecimiento } \\
\text { crecimiento natural negativo }\end{array}$ \\
\hline sociales: & $\begin{array}{l}\text { bajo nivel de vida } \\
\text { falta de infraestructuras/insuficiencias de servicios } \\
\text { desempleo/falta de puestos de trabajo } \\
\text { conflictos entre grupos/intereses económicos } \\
\text { conflictos entre viejos y nuevos pobladores }\end{array}$ \\
\hline económicos: & $\begin{array}{l}\text { pérdida de cosechas por causas meterológicas } \\
\text { excedentes de producción } \\
\text { crisis de algunos cultivos (falta de competitividad) } \\
\text { problemas de comercialización (excedentes/falta } \\
\text { de calidad) } \\
\text { conflictos con otros agricultores } \\
\text { dificultades en la exportación } \\
\text { limitación de la producción (imposición de cuotas) } \\
\text { control de las empresas agroalimentarias }\end{array}$ \\
\hline medioambientales: & $\begin{array}{l}\text { erosión } \\
\text { agotamiento de suelos } \\
\text { incendios } \\
\text { deforestación } \\
\text { contaminación difusa } \\
\text { agotamiento/salinización de acuíferos } \\
\text { deterioro del paisaje natural y cultural }\end{array}$ \\
\hline
\end{tabular}


Cuadro 4. Cuadro conceptual del espacio rural

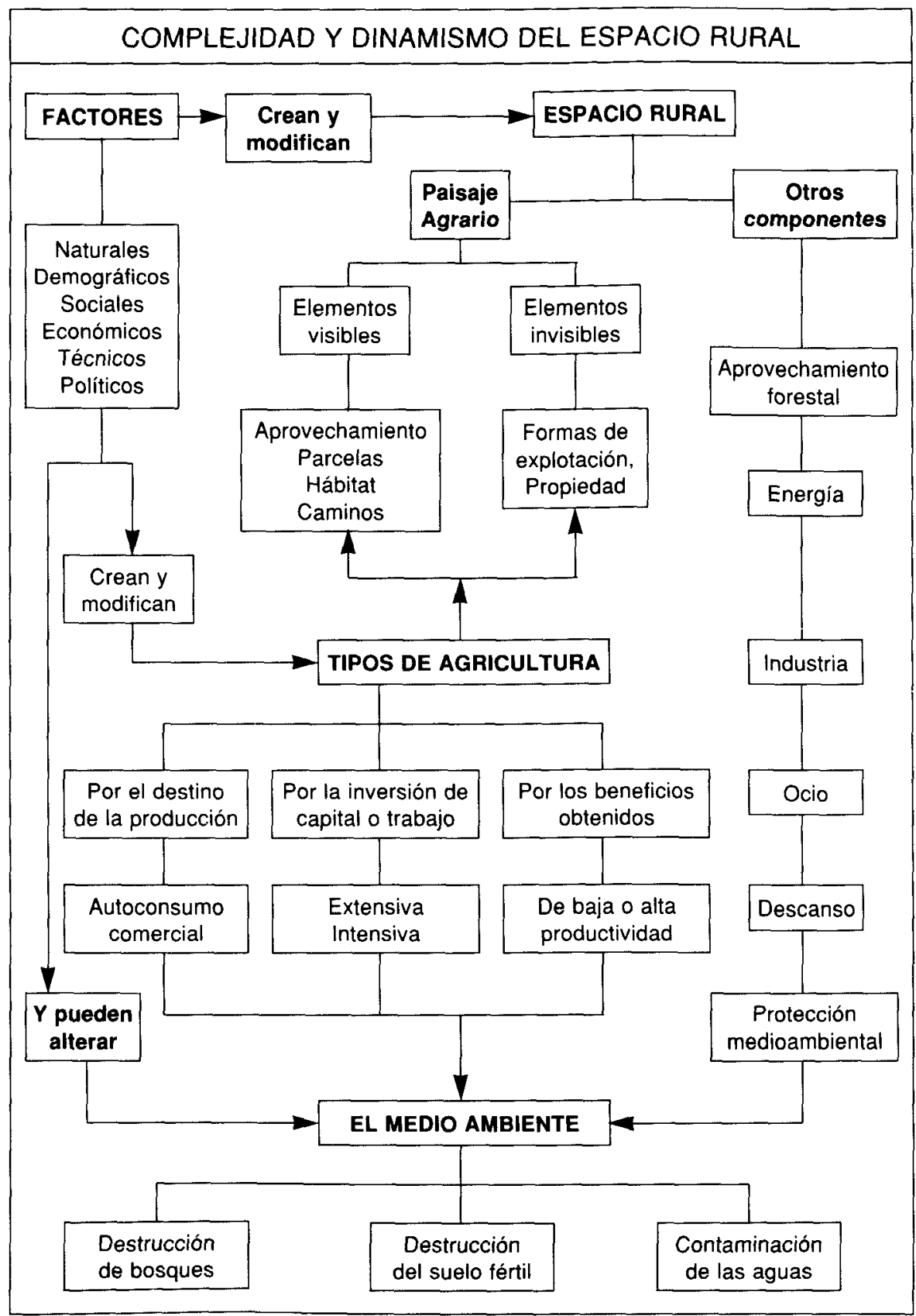


Pero la presentación estática, a base de cuadros estadísticos poco elaborados que no permiten ver los procesos que hay en marcha, debe ser sustituida por un enfoque más dinámico, sobre todo cuando se trate de la Geografía de España de segundo curso de Bachillerato. Para ello hay que prestar mayor atención a todos y cada uno de los factores que originan y hacen evolucionar a los elementos de las estructuras agrarias y al conjunto del espacio rural (Sánchez, J. y Zárate, A., 1989; Rubio, M‥T. y Zárate, A., 1998).

En el análisis de los factores demográficos y sociales hay que aludir a la evolución, características y problemas de la sociedad rural y de la población activa agraria y no agraria. En el tratamiento de los factores naturales se debe introducir el enfoque ecogeográfico que resalta de manera explícita la significación de cada uno de ellos en cuanto a su carácter favorable o desfavorable respecto a los diferentes componentes del espacio rural. Especial atención debe prestarse también a los factores económicos, técnicos y políticos que son los más decisivos para explicar los cambios recientes, coyunturales o definitivos, y la transformación forzada de los componentes del espacio rural; entre ellos, la evolución del sistema económico y de los tipos de agricultura, la difusión de las innovaciones en el mundo rural y la aplicación de políticas agrarias o de desarrollo rural puestas en marcha por organismos estatales o supraestatales.

Junto a esta mayor atención a los factores del espacio rural, con su corolario de limitaciones o potencialidades, el enfoque más integrador y dinámico exige dedicar un lugar a los otros componentes del espacio rural que cada vez adquieren mayor significación desde el punto de vista económico, social, paisajístico y territorial, y que representan las nuevas funciones que muchos de estos espacios empiezan a ejercer en los países desarrollados tras la pérdida de importancia de la actividad agropecuaria; tales son la función residencial, industrial, de ocio, turismo, medioambiental, etc. De esta forma, el espacio rural adquiere el aspecto complejo y vital que hoy le caracteriza y que debe tranmitirse a traves de la educación geográfica.

\section{CRISIS Y TRANSFORMACIONES EN EL MUNDO RURAL: LA CONFIGURACIÓN DE NUEVOS ESPACIOS RURALES}

Un nuevo enfoque en la enseñanza del medio rural, más integrador y dinámico que el utilizado hasta ahora, necesariamente tiene que dar más relevancia a los procesos que se desarrollan en el campo y a los problemas que de ellos se derivan. Las innovaciones técnicas y la integradción 
de la economía agraria en un sistema económico abierto y en un contexto extremadamente competitivo han provocado profundas transformaciones en el mundo rural. En unas áreas la producción ha podido mantener la competitividad, transformando las estructuras agrarias y los sistemas productivos y avanzando hacia la especialización. En otras, la agricultura tradicional no tuvo capacidad para renovarse, hundiéndose ante la presión del mercado y provocando el éxodo rural. Son numerosos los ejemplos de uno u otro signo que podemos observar en todas las regiones españolas (VV.AA., 1980 y 1984; Bosque, 1988; García Delgado, J.L. y Muñoz Cidad, 1988; Cabero y otros, 1992; Gil Olcina, A, y Morales Gil, A. 1993; Ramos Real, E. y Cruz Villalón, J., 1995).

Pero la crisis de los espacios rurales ha sido de tales dimensiones y ha alcanzado a tantas regiones y comarcas agrícolas que en los países desarrollados, y especialemente en la Unión Europea, ha suscitado una honda preocupación entre los responsables de la política regional (COMISIÓN EUROPEA, 1988a y 1988b). Pero, a la vez, los excedentes y los problemas medioambientales ocasionados por la intensificación de la producción agropecuaria ha originado una reacción en contra del productivismo agrario.

Para el caso español, el cuadro $n^{\circ} 5$ muestra los tres modelos de actividad agraria que han tenido lugar y se han sucedido en los últimos cincuenta años, junto con las dos transiciones que el mundo rural se ha visto y se ve obligado a soportar. Las consecuencias han afectado y afectan a la ocupación del suelo, a la producción, a la población activa agraria, a la sociedad rural y a las estructuras productivas.

Consecuencia de todo este complejo proceso en el que se combinan modernización y crisis, mecanización y abandonono, descenso de población y envejecimiento, especialización e integración, desintegración y reorientación, el espacio rural, ya variado por sus formas de adaptación al medio natural, se ha diversificado mucho más; esta vez, debido al grado de incidencia que en cada lugar ha tenido el citado proceso y a la naturaleza de sus consecuencias.

Es de un gran interés didáctico abordar la enseñanza-aprendizaje del mundo rural desde esta perspectiva del cambio y transformación, impuestos por la fuerza imparable de la modernización y globalización económica. Asimismo, se debe poner el acento en los contrastes y desigualdades que ese proceso ha provocado y está provocando en el interior del espacio rural. Así, junto a la clasificación ecogeográfica de los espacios rurales españoles (de la regiones atlánticas, interiores, mediterráneas, insulares, de montaña, etc.), conviene introducir ya en los niveles de la enseñanza 
El espacio rural en la enseñanza secundaria: hacia un enfoque más integrador...

secundaria una nueva tipología basada en los criterios más dinámicos que la perspectiva histórica proporciona.

Cuadro 5. Tres modelos de actividad agraria en España

\begin{tabular}{|c|}
\hline Tres modelos de actividad agraria en España \\
\hline 1. Modelo tradicional: \\
- Intensivo en mano de obra \\
- Poco integrado en sistema económico general \\
Transición 1a \\
- marco nacional \\
- introvertido \\
- protección a la producción \\
- grandes proyectos estatales de infraestructuras \\
2. Modelo productivista: \\
- Intensivo en energía \\
- Fuertemente integrado \\
Transición $2^{a}$ \\
- contexto comunitario \\
- mucho más abierto \\
- apoyo al productor \\
- desarrollo rural amplio \\
3. Modelo postproductivista: \\
- Preocupación por la mayor calidad que demanda el mercado \\
- Basado en las exigencias que impone la nueva valoración del \\
medio ambiente \\
- Producciones menos intensivas \\
\end{tabular}

Una buena propuesta es la que hacen F. Molinero y M. Alario (1994) y que se basa en las distintas situaciones que la citada modernización de la agricultura ha generado en el campo español. Por su dinamismo, por la naturaleza de sus producciones, por el grado de integración económica, por la vitalidad demográfica y por la densidad de las infraestructuras y servicios se pueden diferenciar hasta once tipos de regiones agrarias. En el 
cuadro $\mathrm{n}^{\circ} 6$ se presenta un resumen simplificado de esa propuesta para el nivel de Bachillerato. Los ocho tipos de regiones rurales, agrupadas en cuatro grandes apartados, componen un cuadro suficientemente expresivo de los nuevos espacios rurales configurados en el transcurso de las últimas décadas y que no dejan de tener una estrecha relación con los factores ecogeográficos.

Cuadro 6. Tipos de regiones rurales españolas

Diversidad de espacios rurales en España

A) Espacios rurales dinámicos, con actividad agraria secundaria

3. Areas rurales con implantación del turismo masivo: mutación total de las estructuras agrarias bajo la presión del turismo de masas; en gran parte de las franjas costeras.

4. Franjas periurbanas: mutación total o parcial provocada por la expansión urbana que ocupa el suelo agrario con otros usos y transforma la actividad agraria en función de la demanda urbana.

B) Espacios rurales complejos, con importante actividad agraria

3. Areas de regadios consolidados: modernización de las estructuras y desarrollo de la industria y de los servicios; densidad de población relativamente alta. Ejem.: Riberas del Duero, del Ebro o Cinca-Segre, regadíos mediterráneos o andaluces.

4. Areas de actividad mixta agricola-ganadera: la pluriactividad de sus habitantes, facilitada por las buenas condiciones ecológicas o la variedad de posibilidaes económicas de la comarca, permite la existencia de una densidad superior a 40 habs. $/ \mathrm{km}^{2}$, como ocurre en gran parte de Galicia y algunas comarcas interiores catalanas.

C) Espacios rurales con actividad agropecuaria especializada y extensiva

5. Areas de secano de calidad: exige gran cantidad de mano de obra y la producción se halla bien integrada en el mercado, como ocurre en las campiñas béticas y en las comarcas vitícolas y olivareras.

6. Areas de secanos cerealistas y pastizales extensivos: la gran mecanización y la producción extensiva ha provocado éxodo rural, envejecimiento y bajas densidades de población, como ocurre en la Cuenca del Duero, Penillanura extremeña y Cuenca de Castilla-La Mancha. 
El espacio rural en la enseñanza secundaria: hacia un enfoque más integrador...

Cuadro 6. Tipos de regiones rurales españolas (continuación)

\begin{tabular}{|l|}
\hline Diversidad de espacios rurales en España \\
\hline D) Espacios rurales profundos ( $1 / 4$ parte de territorio español) \\
7. Areas rurales con fuertes desequilibrios demográficos y algunos \\
recursos endógenos: densidades entre 5 y 10 habs $/ \mathrm{km}^{2}$; parte de \\
las penillanuras y algunas comarcas en el interior de las montañas. \\
8. Areas rurales con fuertes desequilibrios demográficos, de impo- \\
sible recuperación: la densidad de población es inferior a 5 \\
habs/km² y la inexistencia de recursos económicos hace casi im- \\
posible su revitalización; son las comarcas de las montañas inte- \\
riores y los piedemonte de transición entre la montaña y el llano.
\end{tabular}

Una clasificación como ésta permite abordar con cierta coherencia el estudio de los procesos de abandono de la actividad agraria tradicional, los procesos de sustitución y transformación radical, los de modernización e integración económica, las nuevas agriculturas y los impactos de la expansión urbana en el espacio rural circundante (Cabero, V., 1998): todo un esquema de trabajo para el estudio de los contrastes regionales del mundo rural, como factor de diferenciación regional y de configuración de los desequilibrios interterritoriales; y una tipología que complementa y enriquece la presentación de los espacios rurales diferenciados por su distinta adaptación a los medios ecológicos.

Se trata de situaciones contrastadas dentro del mismo espacio rural, surgidas como resultado de la reciente evolución histórica que ha sido muy rápida y compleja, afectando a las funciones del mundo rural, a las estructuras agrarias, a la población campesina y a sus modos de vida, a la organización económica y, por supuesto, a los paisajes rurales.

Introducir esta nueva conceptualización del espacio rural, que lleva consigo dinamismo, evolución y diferenciación permanente, sin desplazar por ello a la clasificación ecogeográfica que sigue manteniendo vigencia, supone establecer un esquema organizativo de los distintos contenidos que permite articular de manera lógica un gran número de conceptos, procesos y problemas, aplicados a situaciones concretas de distintos espacios geográficos. Además, al enfoque estático de las morfologías y las estructuras agrarias, muy relacionadas con los factores naturales y con la herencia del pasado, se añadiría el enfoque dinámico de los cambios recientes y actuales producidos por factores técnicos, económicos o políticos. 
Un enfoque como este permitiría con gran lógica prestar mayor atención a los problemas de la sociedad rural, sobre todo en las áreas más deprimidas, introducir la polémica en torno al concepto, naturaleza y tipos de desarrollo rural y de sus diferentes políticas y plantear el debate sobre las consecuencias medioambientales del productivismo agrario. Temas que tanto en el segundo ciclo de la Enseñanza Secundaria Obligatoria (ESO), como en la Geografía de España de segundo curso del nuevo Bachillerato convendria ya desarrollar, si queremos que nuestros jóvenes adquieran un buen conocimiento del mundo real en el que viven y mejoren su grado de sensibilidad hacia los problemas sociales y medioambientales del mundo actual.

\section{EL PAISAJE RURAL, UN GRAN RECURSO DIDÁCTICO}

Debemos conseguir, pues, que el estudio del espacio rural sea más integrador y dinámico y que su aprendizaje en la enseñanza secundaria resulte más atractivo y eficaz. Para ello no debemos olvidar un procedimiento que ayuda extraordinariamente a conseguirlo: la lectura, el análisis e interpretación del paisaje rural. Éste ha de ser necesariamente un método práctico complementario, no alternativo ni sustitutorio al estudio teórico. En realidad, el paisaje es el aspecto o la cara visible del espacio geográfico. La Geografia, como otras disciplinas, lo ha convertido en objeto de estudio; pero enseñar a verlo, sentirlo e interpretarlo debería ser igualmente uno de los principales objetivos de la educación geográfica (Pena, R., 1996), por lo que significa en cuanto a visión integradora de los procesos de ocupación, explotación y transformación del teritorio.

Claro que una lectura del paisaje rural en el segundo ciclo de la ESO y en Bachillerato no puede quedarse en la observación y análisis de los distintos elementos visibles, más propios de la enseñanza de la Geografía en los cursos anteriores. Hay que partir de una buena observación y un completo análisis para entrar de lleno en las claves de la explicación e interpretación. Y esto no es posible sin el estudio teórico previo de los factores, interrelaciones, procesos y problemas que dan vida y dinamismo a los diferentes espacios rurales.

Con el estudio del paisaje rural disponemos de un excelente recurso didáctico que nos va a permitir potenciar la capacidad de observación, repasar y reforzar el aprendizaje de un gran número de conceptos, practicar el método indagatorio para establecer relaciones entre los distintos elementos que aparecen en cada paisaje, comprender la génesis y las transformaciones de los elementos de un paisaje en función de los factores que actúan sobre ellos, y reflexionar en torno a los valores culturales y 
medioambientales a partir de los paisajes observados. Una selección significativa de paisajes ruales españoles puede servirnos de excelente instrumento para comprender las transformaciones históricas y medioambientales del campo español (Cabero, V., 1992 y 1998).

En el cuadro siguiente se presenta esquemáticamente una guía práctica, con elementos de observación y sugerencias para la indagación e interpretación. Puede servir de punto de partida y cada profesor puede desarrollar la actividad hasta el nivel de profundidad que considere posible u oportuno, dadas las peculiaridades de sus alumnos. En cualqueir caso, no se debe limitar a la simple observación y descripción de los elementos visibles, sino que, a partir de ellos, se deben poner de relieve las estructuras geoecológicas y geoeconómicas, para pasar después a la interpretación de las relaciones entre los elementos y a la comprensión de todo el paisaje en su totalidad, contextualizándolo en el tiempo y en un espacio más amplio.

Cuadro 7. Esquema-guía de lectura del paisaje rural

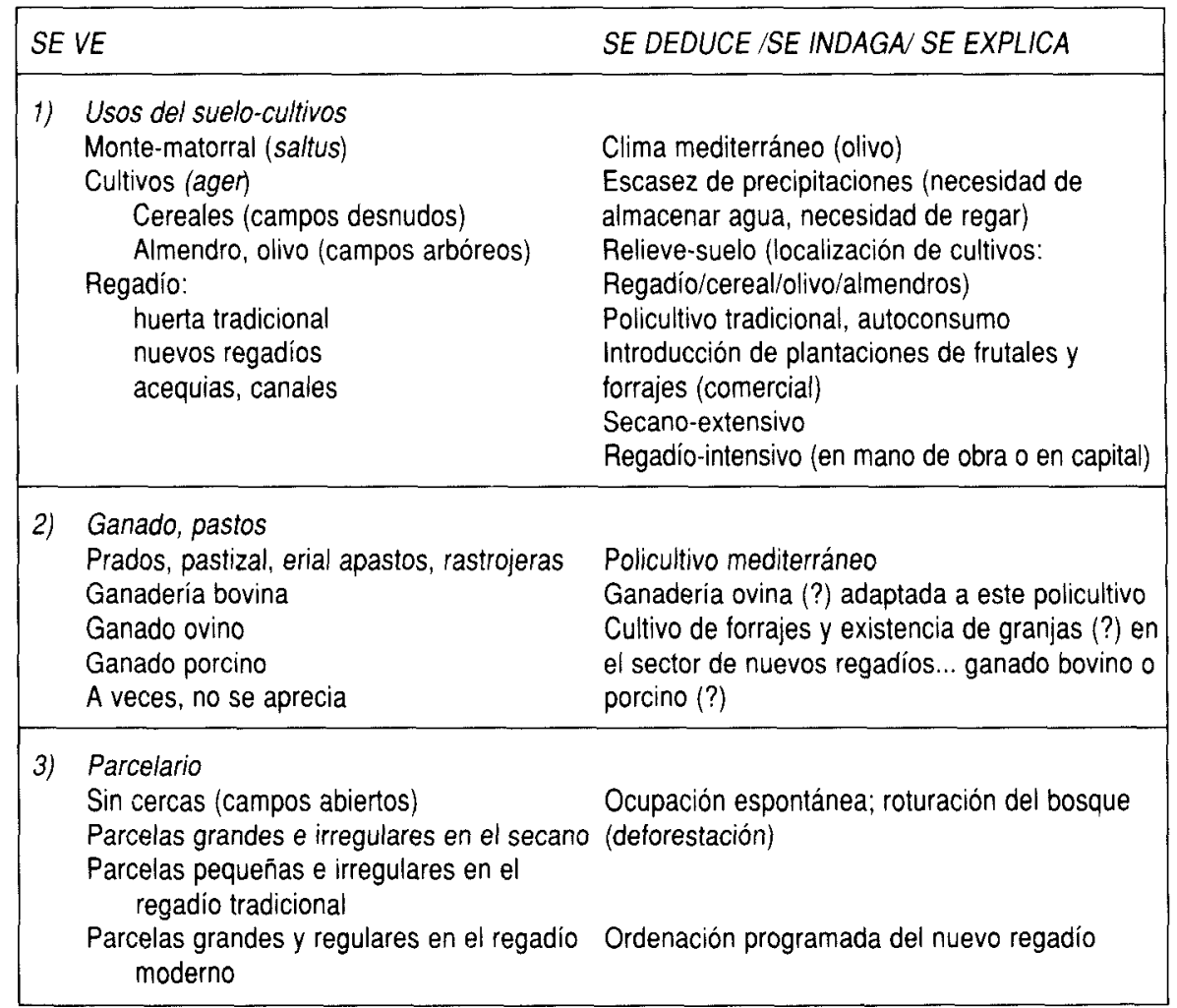


Busouets, J. (1996): "La lectura visual del paisaje. Bases para una metodología". IBER, $n^{\circ}$ 9, Grao, Barcelona, págs. 53-59.

CABERo Diéguez, V. et alt. (1992): El medio rural español: cultura, paisaje y naturaleza (homenaje a $D$. Angel Cabo Alonso). Universidad de Salamanca-Centro de Estudios Salmantinos, Salamanca.

CABero Diéguez, V. (1998): "Los paisajes rurales. Contrastes y procesos de transformación" en Paisaje y Medio ambiente, Universidad de Valladolid, Valladolid, págs. 75-92.

COMISIÓN DE LAS COMUNIDADES EUROPEAS (1988a): El futuro del mundo rural. Editado por IRYDA, MAPA, Madrid, pp.11-138.

COMISIÓN DE LAS COMUNIDADES EUROPEAS (1988b): «Iniciativa Común de Desarrollo Rural (LEADER)", Doc. n L374 de 31-12-88.

Estébanez, J. (1986): "Tendencias en Geografía rural" en Garcia Ballesteros, A.: Teoría y práctica de la Geografía, Alhambra Universidad, Madrid, págs. 225-258.

ESTÉBANEZ, J. (1996): "La geografía, hoy: un reto educativo en el marco de la reforma educativa". III Jornadas de Didáctica de la Geografía, AGE-Grupo de Didáctica de la Geografía, Madrid, págs. 15-29.

Ferrer Rodriguez, A. y Saenz Lofite, M. (1992): “Las actividades agrarias" en Bosque, J. y VILÁ VALENTi, J. (Coord.) (1989-1992): Geografía de España, Planeta, Barcelona, vol. 2, págs. 275-456.

Frutos, Må. L. (1995): “Las actividades rurales» en Carreras, C. y Garcia Ballesteros, A. (Dirs.) (1995): Geografía de España. Edit. Océano, Barcelona, vol. 5, págs. 855-931.

García Delgado, J.L. y MuÑOz CIDAD, C. (1988): "La agricultura: cambios estructurales en los últimos decenios" en Garcia Delgado, J.L. (Dir.): España: Economía. Espasa-Calpe, Madrid, págs. 119-151.

Garcia Ramón, Mz.D.; Julia Pujol, A.F. y Valdovinos Perdices, N. (1995): Geografía rural. Edit. Síntesis, Madrid.

GaRCía RAMÓN, Mª.D. (1992): “Desarrollo y tendencias actuales de la Geografía rural (19801990). Una perspectiva internacional y una agenda para el futuro". VI Coloquio de Geografia Rural. Ponencias, Universidad Autónoma de madrid, págs. 13-35.

GILG, A.W. (1985): An introduction to Rural Geography. Arnold, Londres.

GiL Olcina, A. y MORAles Gil, A. (Edit.) (1993): Medio siglo de cambios agrarios en España. Instituto de Cultura Juan Gil-Albert, Alicante, $884 \mathrm{p}$.

Gómez ORTIz, A. (1996): «El paisaje como elemento de refencia para el estudio de los diversos aspectos geográficos. Una aproximación a su significado curricular ». III Jornadas de Didáctica de la Geografía, A.G.E.-Grupo de Didáctica de la Geografía, Madrid, págs. 195218.

KAISER, B. (1990): La renaissance rurale. Sociologie de campagnes du monde occidental. Armand Colin, Paris.

LÁzARo ARAujo, L. (1995): “El desarrollo rural en el contexto de la Unión Europea» en Ramos Real, E. y CRUz VILLALÓN, J. (Coord) (1995), págs. 237-255.

MOLINERO, F. (1993): “Lógica espacial del sistema productivo: el declive de las actividaddes agrarias y pesqueras" en MENDEZ, R. R. y MOLINERO, F. (Coord.) (1993): Geografía de España. Edit. Ariel, Barcelona, págs. 324-378.

MOLINERO, F. y ALARIO, M. (1994): “La dimensión geográfica del desarrollo rural: una perspectiva histórica". Revista de Estudios Agrosociales, $n^{0} 169$, págs. 53-87.

PENA VILAR, R. (1996): "Geografia, paisaje y educación ambiental». III Jornadas de Didáctica de la Geografía, A.G.E.-Grupo de didáctica de la Geografía, Madrid, págs. 323-328.

PUENTE, L. DE LA (1998): “Viejos y nuevos enfoques en el estudio del medio rural». Iber, $n^{0}$ 16, págs. $45-54$.

Ramos Real, E. y Cruz Villalón, J. (Coord) (1995): Hacia un nuevo sistema rural. M.A.P.A., Madrid.

RIvAS, J. (1992): "El estudio preliminar de un paisaje». II Congreso de Ciencia del Paisaje, vol. 1, págs. 187-193.

RUBIO, Ma .T. y ZÁRATE, A. (1998): Del espacio agrario al espacio rural. Video, UNED, Madrid. SABATER, F. (1998): “Potenciar la razón" en El País, 8-X|I-1998, págs. 31-32

SÁENZ LORITE, M. (1988): Geografía agraria. Introducción a los paisajes rurales. Edit. Síntesis, Colecc. Geografía de España, n² 7, Madrid, 157 p. 
Sánchez OgallaR, A. (1998): "La observación e interpretación del paisaje". Didáctica Geográfica, Segunda época, $n^{9} 2$, Grupo de Didáctica de la Geografía de la A.G.E., págs. 45-55.

SÁNCHEZ SÁNCHEZ, J. (1994): “El paisaje rural como recurso didáctico: de la observación a la comprensión". II Congreso de Ciencia del Paisaje. Barcelona, vol. 2, págs. 533-542.

SANCHEZ SANCHEZ, J. (1996): "Pluriactividad y paisaje agrario en la montaña mediterránea". VIII coloquio de Geografía Rural, Jaca-Zaragoza, págs. 379-390.

SÁNCHEZ, J. y ZARATE, A. (1989): El mundo en que vivimos. Geografía $2^{\circ}$ de Bachillerato. Edit. S.M., Madrid.

SAntos, M. (1996): De la totalidad al lugar. Oikos-Tau, Barcelona, 167 p.

SoURP, R. (1995): "L'analyse du paysage, une didactique pour la géographie". L'information géographique, $\mathrm{n}^{2} 4$, pp170-175.

Tio, C. (1995): "Implicaciones de la nueva Política Agraria Internacional para el sistema agrario español” en Ramos Real, E. y Cruz Villallón, J. (Coord) (1995), 157-167.

VV. AA. (1984): Coloquio hispano-francés sobre espacios rurales. MAPA, Madrid, 2 vols.

VV. AA. (1980): Los paisajes rurales de España. AGE, Valladolid, 356 p. 